${ }^{1}$ Universitätsklinik für Allgemeine Innere Medizin, Inselspital Universitätsspital Bern

${ }^{2}$ Berner Fachhochschule Gesundheit

${ }^{3}$ Inselspital Universitätsspital Bern

${ }^{4}$ Institut für Pflegewissenschaft, Universität Basel

Yvonne Stauffer $^{1,2}(\mathrm{MSCN})$, Elisabeth Spichiger ${ }^{3,4}$ (RN, PhD), Claudia Mischke (Prof. Dr. rer. medic., MPH)

\title{
Komplexe Medikamentenregime bei multimorbiden älteren Menschen nach Spitalaufenthalt - eine qualitative Studie
}

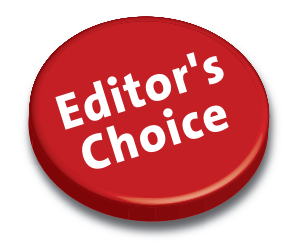

- Was ist (zu dieser Thematik) schon bekannt?

Komplexe Medikamentenregime nach Spitalaufenthalt stellen eine Herausforderung dar.

- Was ist neu?

Das selbstständige Medikamentenregime ist für ältere Menschen wichtig, aber fehlendes Wissen der Gesundheitsfachpersonen zur Lebenssituation, ungenaue Informationen und Probleme im Medikamentenbezug erschweren die korrekte Einnahme zu Hause.

- Welche Konsequenzen haben die Ergebnisse für die Pflegepraxis? Um die Kontinuität über die Spitalzeit hinaus zu fördern, werden lebensweltnahe Konzepte zum Medikamentenregime benötigt.

Hintergrund: Ältere Menschen sind oft multimorbid und haben dadurch ein komplexes Medikamentenregime, das sich bei Spitalaustritt ändert und für sie herausfordernd sein kann. Die Sicht älterer Menschen im Umgang mit dem Medikamentenmanagement, vor allem nach Spitalaustritt, wurde bisher kaum erforscht.

Fragestellung: Diese Studie geht der Frage nach, wie multimorbide ältere Menschen die Einnahme einer Vielzahl von Medikamenten nach Spitalaustritt erleben und wie sie damit umgehen.

Methode: Ein qualitativ-deskriptives Verfahren wurde gewählt, zehn Interviews durchgeführt. Die Daten wurden offen in Anlehnung an Saldaña (2013) in zwei Zyklen codiert.

Ergebnisse: Für ältere Menschen sind trotz Multimorbidität das Bewahren der Selbstständigkeit und die Verant-

Manuskript eingereicht am 26.11.2013 Manuskript akzeptiert am 10.4.2014 wortungsübernahme für die Medikamenteneinnahme wichtig. Durch jahrelange Einnahme eignen sie sich Routine im Umgang mit Medikamenten an, neue Verordnungen werden in die Alltagsroutine integriert. Ungenaue Informationen der Gesundheitsfachpersonen und Schwierigkeiten der Befragten, die Medikamente nach Spitalaustritt zu beschaffen, können dazu führen, dass die medikamentöse Therapie zu Hause nicht korrekt und lückenlos weitergeführt wird. Vertrauensperson ist, auch für Informationen zur medikamentösen Therapie des Spitals, der Hausarzt/die Hausärztin. Schlussfolgerungen: Es empfiehlt sich, im Spital eine den Bedürfnissen der älteren Menschen angepasste Beratung durchzuführen, in der die medikamentöse Therapie, ihre Integration in den Tagesablauf und mögliche Lücken im Medikamentenmanagement für die Zeit nach dem Austritt besprochen werden.
Schlüsselwörter: Ältere Menschen, Polypharmazie, Selbstmanagement, Medikamentenregime nach Spitalaustritt, qualitative Studie

\section{Einleitung}

Im Jahr 2012 waren in der Schweiz 17,4\% der Bevölkerung über 65-jährig (Bundesamt für Statistik [BfS], 2014a). Das Alter von 65 Jahren wird vor allem aus soziologischer Perspektive als Übergang in die Altersphase angeführt und mit dem Beginn der postproduktiven Phase verknüpft. Andere theoretische Ansätze fokussieren mehr auf biologische oder entwicklungspsychologische Alterungsprozesse, wobei hier die Grenzen zwischen verschiedenen Altersphasen schwer definierbar sind (Wurm, Wiest \& Tesch-Römer, 2010). Als ältere Menschen werden in dieser Studie in Anlehnung an das soziologische Verständnis von Alter daher Menschen im Ruhestand (gewöhnlich ab 65 Jahre) verstanden (Wurm et al., 2010; Göckenjan, 2010).

Eine Literaturanalyse zu Alter und Multimorbidität zeigt, dass je nach Studie 55 bis $98 \%$ der über 65-jährigen Menschen multimorbid sind (Marengoni et al., 2011). Demografische Prognosen gehen davon aus, dass, bedingt durch die steigende Lebenserwartung, die Zahl der älteren Menschen mit Multimorbidität weiter zunehmen wird (BfS, 2010). Boyd und Fortin (2010) definieren Multimorbidität als Koexistenz von zwei oder mehr chronischen Krankheiten, wobei die eine nicht zwingend bedeutsamer als die andere ist. Mit der Multimorbidität steigt die tägliche Medikamentenmenge (Häfeli, 2011; Neuner-Jehle, 2011) und gleichzeitig die Gefahr von fehler- 
haften Einnahmen (DeBartolomeo und das Mitentscheiden-Können wichMager \& Madigan, 2010).

Aus Studien zum Medikamentenmanagement liegen Erkenntnisse zur Adhärenz von Patient(inn)en und zu Faktoren vor, welche die Adhärenz beeinflussen. Allerdings beschreiben diese mehrheitlich die Sicht der Gesundheitsfachpersonen (Swanlund, Scherck, Metcalfe \& Jesek-Hale, 2008). Banning (2009) und Ludwig (2005) zeigen in Literaturreviews, dass das Erleben der Patient(inn)en im Umgang mit ihren Medikamenten bislang kaum untersucht wurde. Erst in den letzten Jahren gewinnt die Perspektive der Betroffenen an Bedeutung. Diskontinuitäten im Medikamentenmanagement können unter anderem den Krankheitsverlauf negativ beeinflussen, zu häufigeren Arztkontakten oder Rehospitalisationen führen und ziehen so sozio-ökonomische Konsequenzen nach sich (Swanlund et al., 2008). Vorliegende Studien fokussieren auf chronisch kranke Erwachsene aller Altersklassen mit einer spezifischen Erkrankung. Zur Bewältigung des komplexen Medikamentenmanagements werden folgende Faktoren von den Befragten als relevant betrachtet: subjektiver Gesundheitszustand und Krankheitsverständnis, Routine im Umgang mit Arzneimitteln, Integration in den Alltag, Wissen zu Wirkung und Nebenwirkung, Zugang zu unterstützenden Organisationen wie spitalexterne Krankenpflege (Gordon, Smith \& Dhillon, 2007; Haslbeck, 2008; Reid et al., 2005). Nur wenige Studien fokussieren auf das Erleben der Medikamenteneinnahme multimorbider älterer Menschen. Entweder werden ältere Menschen mit einer spezifischen Erkrankung befragt oder es fehlen Angaben zum Krankheitszustand. Die Ergebnisse zeigen, dass das Erstellen eines Erinnerungssystems und das Erlangen einer Routine in der Medikamenteneinnahme ebenso wie das Selbstmanagement tig sind (Lumme-Sandt \& Virtanen, 2002; Ybanez-Blomstrom, 2008). Der Alltag wird durch das Medikamentenmanagement und insbesondere durch Nebenwirkungen von Medikamenten beeinflusst (Kippen, Fraser \& Ellis, 2005). Anfangs steht der Nutzen im Vordergrund, das Erinnern an die Einnahme sowie die Medikamenteneinnahme selbst wird problemlos erlebt. Mit der Zeit wird der Nutzen hinterfragt und je nach Vertrauen ins Gesundheitssystem das Medikamentenregime besser oder schlechter befolgt (Moen et al., 2009).

Erschwert wird die Fortsetzung des Medikamentenmanagements durch Unterbrechungen in Routinen oder Veränderungen im Medikamentenplan (Haslbeck \& Schaeffer, 2009; De Geest, Abraham, Gemoets \& Evers, 1994). Spitalaufenthalte können hier ursächlich sein.

In der Schweiz entfielen 2012 zirka $30 \%$ der stationären Krankenhausfälle auf 70-Jährige und Ältere (BfS, 2014b). Während der Hospitalisation wird die Medikation bei diesen Patient(inn)en fast täglich geändert, in der Folge weicht das Medikamentenregime bei Austritt im Vergleich zum Eintritt ab (Knight, Thompson, Mathie \& Dickinson, 2011). Eine zusätzliche Schwierigkeit bildet die Umstellung der angestammten Medikamente auf Generika mit anderen Namen und anderem Aussehen (Pruszydlo, Quinzler, Kaltschmidt \& Haefeli, 2008). Entsprechend gewinnen aus Sicht der Gesundheitsfachleute die Austrittsplanung und die Unterstützung zu Hause nach Spitalaustritt an Bedeutung. Schwerpunkte bilden Beratungen durch Gesundheitsfachleute, individuelle Interventionen, Unterstützung durch Angehörige und verständliche Austrittsdokumente (Laugaland, Aase \& Barach, 2012). Ryan et al. (2012) betonen in den Schlussfolgerungen ihres
Cochrane Reviews zur patient(inn) enzentrierten Unterstützung in der Medikamenteneinnahme aber, dass eine große Forschungslücke hinsichtlich evidenzbasierter Interventionen für multimorbide Patient(inn)en mit einer Vielzahl von Medikamenten besteht.

Grund für diese Lücke kann die fehlende Wissensbasis sein, denn dem Prozess des Medikamentenmanagements aus der Perspektive multimorbider älterer Menschen wurde bisher kaum Beachtung geschenkt. Dies gilt insbesondere auch für die unmittelbare Zeit nach Unterbrechungen im Alltag beispielsweise durch Spitalaufenthalte. Eine Studie aus England von Knight et al. (2011) beschreibt die Erfahrungen von multimorbiden älteren Menschen und ihrer Angehörigen mit dem Medikamentenmanagement in der ersten Zeit nach Spitalaustritt. Die Ergebnisse zeigen die Unzufriedenheit der Betroffenen mit der Austrittsplanung bezüglich des Medikamentenmanagements auf: Klare schriftliche Informationen, die richtige Medikamentenabgabe für den Austritt sowie eine gute Kommunikation zwischen Ärzt(inn)en im Spital und in den Praxen wären aus Betroffenensicht wichtig. Auch eine amerikanische und eine niederländische Untersuchung stellen das Bedürfnis nach Medikamenteninformationen als zentral heraus (Borgsteede, KarapinarÇarkit, Hoffmann, Zoer \& Van den Bemt, 2011; Foust, Vuckovic \& Henriquez, 2012). Offen bleibt, ob diese Ergebnisse auf die Schweiz übertragbar sind, da beispielsweise die medikamentöse Versorgung bei Spitalaustritt schweizweit unterschiedlich gehandhabt wird, und möglicherweise auch die Information über Medikamentenveränderungen anders erfolgt (MüllerBühl, Gerold, Engeser \& Szecsenyi, 2009). Swanlund et al. (2008) führen ergänzend Unterschiede in kulturellen Wertvorstellungen zu Gesundheit und 
Krankheit, nationalen Gesundheitssystemen und der Verfügbarkeit von Gesundheitsressourcen als problematisch an.

Die vorliegende Studie will daher Erkenntnisse zum Prozess des Medikamentenmanagements in der Zeit nach Spitalaustritt aus Sicht der Betroffenen generieren und untersuchen wie multimorbide ältere Menschen in der deutschsprachigen Schweiz die komplexe und zum Teil neue Medikamententherapie erleben und wie sie damit umgehen.

\section{Methode}

Ziel dieser Forschung war, die Lebenswelt der älteren Menschen aus ihrer Sicht bezogen auf ihr persönliches Medikamentenmanagement $\mathrm{zu}$ beschreiben, um ein besseres Verständnis ihrer sozialen Wirklichkeit zu gewinnen. Daher wurde ein qualitatives deskriptiv-exploratives Design gewählt. Dies erlaubte es, über mehrere Kodierungsphasen bedeutungsvolle Inhalte aus dem Datenmaterial heraus $\mathrm{zu}$ kristallisieren und benennen $\mathrm{zu}$ können (Ritchie \& Lewis, 2003; Sandelowski, 2010).

\section{Teilnehmende und Rekrutierung}

In die Untersuchungsgruppe wurden über 65-jährige multimorbide ältere Menschen, die mehr als drei verschiedene Medikamente pro Tag einnahmen und kurz zuvor hospitalisiert waren, eingeschlossen. Die Teilnehmenden mussten ihr Medikamentenmanagement zu Hause ohne Unterstützung durch Angehörige oder spitalexterne Krankenpflege durchführen. Im Vergleich zum Spitaleintritt musste sich das Medikamentenregime geändert haben. Ausgeschlossen wurden gemäß Angaben im Patient(inn)endossier, Personen, die nicht Deutsch sprachen oder kognitiv beziehungsweise psy- chisch nicht in der Lage waren, ein Interview zu führen. Die älteren Menschen wurden auf fünf Stationen einer Universitätsklinik für allgemeine innere Medizin kurz vor Austritt um ihre Teilnahme gebeten. Mit Personen, die einer Teilnahme zustimmten und die Einverständniserklärung unterschrieben, wurde ein Interviewtermin für die Zeit der ersten vierzehn Tage nach Entlassung vereinbart. Dieser Zeitraum wurde gewählt, damit die älteren Menschen sich an das Erleben in der Zeit unmittelbar nach Spitalaustritt erinnern konnten. Die Interviews erfolgten im häuslichen Umfeld der Teilnehmenden. Aus pragmatischen Gründen wurde die Zahl der Teilnehmenden auf zehn begrenzt. Die Durchführung der Studie einschließlich der Einhaltung ethischer Grundsätze wurde vom Internal Review Board des zuständigen Spitals am 11.10.2012 genehmigt.

In der Klinik erfolgte während der Hospitalisation keine gezielte Beratung zum Medikamentenmanagement. Die Rezeptabgabe mit Medikamentenplan fand am Austrittstag durch die Ärztin/ den Arzt statt, Medikamente wurden nicht nach Hause mitgegeben. Zu Hause mussten anhand des Medikamentenplans aus dem Medikamentenbestand gleichbleibende Medikamente aussortiert und neue in der Apotheke oder beim Hausarzt/bei der Hausärztin bezogen werden.

\section{Datensammlung}

Die Datensammlung erfolgte mittels semistrukturierter Expert(inn)eninterviews im Zeitraum zwischen Oktober und Dezember 2012. Mit Expert(inn) eninterviews kann es gelingen, soziale Sachverhalte, Handlungen und Wissen zu einem bestimmten Untersuchungsgegenstand zu rekonstruieren (Gläser \& Laudel, 2009). Multimorbide ältere Menschen wurden in diesem Sinne als Expert(inn)en ihres persönlichen Medikamentenmanagements betrachtet.
Die Interviews mit ihnen als Expert(inn) en sollten einen Einblick in ihre soziale Wirklichkeit erlauben.

Basierend auf den im Forschungsstand beschriebenen aktuellen Erkenntnissen, wurden für den Leitfaden die Themen aufgegriffen, die sich mit dem Aspekt rund um das Erleben und den Umgang mit Medikamenten in der Zeit nach Spitalaustritt befassten. Ziel war es, die subjektive Sichtweise ebenso wie das implizite Wissen zum eigenen Medikamentenmanagement der Interviewten zu explorieren (Flick, 2011). Die Teilnehmenden stellten ihre aktuellen Medikamente vor und wurden danach mittels offener Fragen nach Erfahrungen zum Medikamentenmanagement nach Spitalaustritt gefragt. Weitere Themen bildeten ihre subjektive Wahrnehmung zur Medikamenteninformation im Spital und die individuelle Bedeutung, die sie den Medikamenten zuschrieben. Dies ermöglichte einerseits eine Strukturierung nach thematischen Inhalten und anderseits viel Spielraum in den Frageformulierungen (Flick, 2011; Gläser \& Laudel, 2009).

Die Interviews wurden in Schweizerdeutsch durchgeführt, digital aufgenommen und pseudonymisiert ins Schriftdeutsche transkribiert. Zusätzlich wurden soziodemografische Daten erfasst sowie medizinische dem Patient(inn)endossier entnommen.

\section{Datenanalyse}

Um das Medikamentenmanagement aus der Perspektive älterer multimorbider Menschen zu erfassen, wurde für die Datenanalyse ein induktiv-offenes Vorgehen gewählt. Dieses Verfahren ermöglichte es, offen an das Datenmaterial heranzugehen, um das Neue und Unerwartete in den Daten entdecken zu können (Miles \& Huberman, 1994; Creshwell, 2007). Der Prozess des Kodierens wurde hierbei in Anlehnung an Saldaña (2013) nicht als ein Verfahren 
verstanden, das einer spezifischen Methodologie zugeordnet ist, sondern als Methode, die das Organisieren und Zuordnen von Daten in Kategorien ermöglicht (Saldaña, 2013). In verschiedenen Kodierungsphasen sollte symbolisch Zusammenfassendes, Auffälliges und Wesentliches identifiziert werden. In Feldnotizen und Memos wurden zudem Analyseschritte und Reflektionen erfasst und in die unterschiedlichen Kodierprozesse einbezogen.

Um Antworten auf die Forschungsfrage zu finden, wurden im ersten Analysezyklus vor allem folgende von Saldaña (2013) beschriebene CodingVerfahren angewendet: Attribut-, Emotion-, Wert- sowie deskriptives Coding. Daten nach Attributen wie soziodemografische Daten, Medikamente vor und nach der Hospitalisation, Diagnosen und Aufenthaltsdauer, zu durchleuchten, half, die Stichprobe zu charakterisieren. Das AttributeCoding gehört daher zum wichtigen Bestandteil in allen qualitativen Forschungen. Um relevante Aspekte und Themen im Zusammenhang mit dem Medikamentenmanagement aus der Perspektive der Betroffenen zu identifizieren, wurde außerdem ein deskriptives Coding durchgeführt, beispielsweise Ausführungen der Patient(inn)en, die den Umgang mit den Medikamenten und verschiedenen Krankheiten beschrieben. Aussagen zu Emotionen, Wertvorstellungen und Einstellungen im Zusammenhang mit der Medikamenteneinnahme wurden als Emotion- und Werte-Codes codiert. Saldaña (2013) hält die Anwendung von Emotion-Coding für geeignet, wenn es um das Beschreiben von Emotionen im Hinblick auf intra- oder interpersonelle Erfahrungen oder Handlungen geht. Dadurch sollte ein besserer Einblick in die Perspektive der Betroffenen und die Lebensumstände, in denen sie ihr Medikamen-

Tabelle 1: Überblick über die Medikamenteneinnahme vor und nach Spitalaufenthalt.

\begin{tabular}{|lcrll|}
\hline Patient(in) & \multicolumn{2}{l|}{$\begin{array}{l}\text { Vor Spitaleintritt: } \\
\text { Anzahl Medikamente und } \\
\text { Häufigkeit der Einnahme }\end{array}$} & $\begin{array}{l}\text { Nach Spitalaustritt: } \\
\text { Anzahl Medikamente und } \\
\text { Häufigkeit der Einnahme }\end{array}$ \\
\hline 1 & 7 & 3-mal täglich & 9 & 4-mal täglich \\
2 & 8 & 3-mal täglich & 7 & 3-mal täglich \\
3 & 7 & 3-mal täglich & 9 & 4-mal täglich \\
4 & 4 & 2-mal täglich & 7 & 4-mal täglich \\
5 & 4 & 3-mal täglich & 6 & 4-mal täglich \\
6 & 7 & 2-mal täglich & 12 & 4-mal täglich \\
7 & 14 & 2-mal täglich & 10 & 2-mal täglich \\
8 & 8 & 6-mal täglich & 8 & 6-mal täglich \\
9 & 3 & 3-mal täglich & 4 & 3-mal täglich \\
10 & 4 & 2-mal täglich & 4 & 2-mal täglich \\
Durchschnitt & 6,6 & & 7,6 & \\
\hline
\end{tabular}

tenmanagement durchführten, gelingen.

Mit dem Werte-Coding sollte ergänzend die Relevanz, die die Betroffenen dem Medikamentenmanagement $\mathrm{zu}$ ordneten, ihre Einstellungen hierzu und ihr Glaube/ihre Einschätzung hinsichtlich des persönlichen Nutzens herauskristallisiert werden. Mit dem Ziel, Hauptthemen zum Medikamentenmanagement aus der Perspektive der Betroffenen zu identifizieren, wurden die Codes im zweiten Zyklus hinsichtlich erkennbarer Muster analysiert. In Anlehnung an Miles und Huberman (1994) wurde zum Beispiel nach Regeln, Gewohnheiten, Gründen und Erklärungen in den Daten gesucht sowie Verbindungen zwischen den Codes herausgearbeitet. Die beiden Zyklen wurden mehrere Male wiederholt, bis die einzelnen Codes und Kategorien sich klar voneinander abgrenzten. Die Daten wurden mithilfe des Softwareprogramms MAXQDA $^{10}$ ausgewertet. Datenerhebung und -analyse erfolgten parallel. Neue Themen, die in den Interviews zur Sprache kamen, wurden im Leitfaden aufgenommen und in den nachfolgenden Interviews weiterverfolgt.

\section{Ergebnisse}

Von zwölf angefragten älteren Menschen nahmen sechs Frauen und vier Männer an der Studie teil. Zwei lehnten eine Studienteilnahme aus Zeitgründen ab. Die Interviews dauerten zwischen 40 und 70 Minuten. Fünf ältere Menschen waren verwitwet, zwei davon hatten einen Lebenspartner, lebten aber alleine, fünf waren verheiratet. Die Frauen waren durchschnittlich älter (82,2 Jahre) als die Männer (72,2 Jahre). Acht von zehn Teilnehmenden hatten eine Berufsausbildung und gaben an, bis zur Pensionierung ihren Beruf ausgeübt zu haben. Die Betagten hatten vier bis neun Hauptdiagnosen. Am häufigsten litten sie an zerebrovaskulären Verschlusskrankheiten, Kardiopathie, arterieller Hypertonie und Diabetes mellitus. Nebendiagnosen waren unter anderem Niereninsuffizienz, maligne Tumorerkrankungen und depressive Episoden. In der nachfolgenden Tabelle 1 finden sich Informationen zur Medikamenteneinnahme vor und nach Spitalaufenthalt.

In der Analyse der Daten kristallisierten sich fünf zentrale Kategorien zum 
Erleben und Umgang der älteren Menschen mit der Medikamenteneinnahme nach Spitalaustritt heraus (Tabelle 2). Durch alle Kategorien hindurch zieht sich das Bedürfnis nach dem Erhalt der Selbstständigkeit. Die Ergebnisse zu diesem Aspekt wurden jeweils themenspezifisch den fünf Kategorien zugeordnet.

Multimorbidität - Erleben und Umgang Das individuelle Erleben und der Umgang mit ihrer Multimorbidität prägen das Medikamentenmanagement der älteren Menschen. Besonders der Eigenständigkeit, trotz krankheitsbedingter Einschränkungen, messen sie eine hohe Bedeutung bei. Äußerst wichtig ist für sie das selbstständige Managen der Multimorbidität, zu dem neben den gewissenhaften Kontrollen von Blutdruck, Blutzucker und Gewicht, das Organisieren, Einhalten von Terminen bei Ärzt(inn)en und Therapeut(inn)en und das Verwalten der Krankenakte auch die selbstständige Medikamenteneinnahme gehören. Nur so können sie die für sie essenzielle Autonomie im Alter bewahren. «Es ist für mich sehr wichtig. (...) Ja in allem, ich bin sehr selbstständig. Da gehört auch das Tablettennehmen dazu» (I.9: 107 - 110).

Krankheiten gehören für die älteren Menschen zum Alter, die sie annehmen

Tabelle 2: Übersicht über Kategorien und Subkategorien.

\begin{tabular}{|ll|}
\hline Kategorien & Subkategorien \\
\hline Multimorbidität - Erleben und Umgang & \\
$\begin{array}{l}\text { Einstellung zu Medikamenten und } \\
\text { deren Bedeutung }\end{array}$ & \\
$\begin{array}{l}\text { Verantwortung für die Medikamenten- } \\
\text { einnahme }\end{array}$ & Zu Hause \\
Im Spital \\
& Kennen der Medikamente \\
& und deren Wirkung \\
& Richten der Medikamente \\
& Erhalt und Wiedererlangen von Routinen \\
$\begin{array}{ll}\text { Unterstützung durch Ärzte/Ärztinnen } \\
\text { und Pflegefachpersonen }\end{array}$ & bei der Medikamenteneinnahme \\
\hline
\end{tabular}

und mit deren Auswirkungen sie lernen umzugehen, auch wenn es ihren Alltag beeinträchtigt. Die Medikamenteneinnahme gehört $\mathrm{zu}$ diesen Einschränkungen, entsprechend ist das Erleben und der Umgang mit der Multimorbidität eng mit der Einstellung zu Medikamenten verknüpft.

\section{Einstellung zu Medikamenten und deren Bedeutung}

Für die älteren Menschen gehören Medikamente zum Alter. Sie sind dankbar, dass sie Linderung bringen und Sicherheit vermitteln. Medikamente empfinden sie als lebenswichtig. Einige hinterfragen die medikamentöse Therapie, sind sich aber der Bedeutung bewusst. Dies prägt ihre Einstellung zu den Medikamenten: «Manchmal frage ich mich, ist dies nötig. Aber jetzt habe ich gesehen, dass es nötig ist. Diese kleine weiße Tablette, die kann mir das Leben retten» (I.10: 180). Andere nehmen die medikamentöse Therapie als gegeben an. «Dies gehört einfach dazu, es bleibt mir nichts anderes übrig» (I.7: 83). Die Bedeutung, welche die Befragten ihren Medikamenten beimessen, verknüpft mit dem Wunsch nach Selbstständigkeit im Alter, zeigt sich auch im Bedürfnis nach Eigenverantwortung bezüglich der Medikamenteneinnahme. 
wäre es mir gleich gewesen. Aber so ist es doch einfacher für sie» (I.7: 115).

\section{Medikamentenmanagement}

Die älteren Menschen schilderten sehr differenziert und detailliert die Prozesse rund um ihr komplexes Medikamentenmanagement. Dies umfasst das Wissen um Wirkungen und Nebenwirkungen, das Organisieren und Richten der Medikamente ebenso wie das Entwickeln von Routinen und Erinnerungsstrategien für die Medikamenteneinnahme. Die Verantwortungsübernahme für diese Prozesse spiegelt auch hier das Bedürfnis nach Selbstständigkeit wider.

Kennen der Medikamente und deren Wirkung: Alle älteren Menschen nehmen seit Jahren mehrere Medikamente ein, kennen deren Wirkung, Aussehen und Namen. Auch bei neu verordneten Medikamenten sind Wirkung und Namen überwiegend bekannt. Der Umgang mit Generika bereitet ihnen keine Schwierigkeiten. Als Hilfe schreiben einige den Namen des Originalpräparats auf die Schachtel des Generikums. Einige ältere Menschen lesen die Beipackzettel zur Wissenserweiterung genau durch. «Die, die ich kenne, schaue ich nicht immer wieder nach, aber die, die ich nicht kenne, da lese ich alles nach» (I.1: 72). Mögliche Nebenwirkungen nehmen sie zur Kenntnis, lassen sich aber dadurch nicht verunsichern. «Wenn man das alles sieht, dann hat man nur Nebenwirkungen. Man muss sich auch nicht selbst verrückt machen» (I.5: 128). Bei Wissen um Nebenwirkungen befürchten einige ältere Menschen, diese eher zu spüren. Die Medikamente müssen aber trotzdem eingenommen werden. «Da könnte man schon etwas in Panik kommen. Darum habe ich dies nicht mehr gemacht» (I.3: 121). Andere lesen die Prospekte aus diesen Gründen nicht. Die nötigen Informationen erhalten sie vom Hausarzt, von der Hausärztin.
Richten der Medikamente: Die älteren Menschen verfügen über unterschiedlichste Methoden, mit oder ohne Hilfsmittel, um ihre Medikamente zu richten: Einige richten die Medikamente vor jeder Einnahme, andere ihren Wochendispenser immer zur selben Zeit nach genau definiertem System. Sie nehmen sich Zeit, brauchen Ruhe und wollen nicht gestört werden. «Und dann sitze ich an den Schreibtisch und muss mir richtig Zeit nehmen» (I.4: 84). Schwierig ist das Anpassen des Inhalts des Wochendispensers nach Veränderung der Verordnung. Gestoppte Medikamente nehmen die Betroffenen mühselig mit einer Pinzette aus den Fächern. Schwer fällt, das richtige Medikament aus den oft ähnlichen weiBen Tabletten herauszusuchen: «Als ich heimgekommen bin, da musste ich die neuen Medikamente richten. Da hatte ich einen Moment einen Knopf, mit denen, die noch drin waren. Das Aspirin musste ich herausnehmen und eine Schmerztablette rein tun, beide waren wei $\beta$ » (I.4: 181).

Die älteren Menschen nutzen verschiedenste Orientierungshilfen: Etiketten auf den Originalpackungen mit zusätzlichen, selbst angebrachten Markierungen, zum Teil selbst verfasste Medikamentenübersichtspläne. Sie richten ihre Medikamente auch aus dem Gedächtnis heraus. «Ja, ich habe dieses Papier, aber ich brauche es gar nicht» (I.2: 56).

Erhalt und Wiedererlangen von Routinen bei der Medikamenteneinnahme Die älteren Menschen beschreiben die jahrelange Routine in der Medikamenteneinnahme. Sie betonen, dass die Einnahme routiniert sein muss. Wenn man «daran denken müsse», bestünde eine große Gefahr des Vergessens. Die Routine ist oft an eine Alltagstätigkeit gekoppelt. «Am Abend, wenn im Fernsehen Reklame ist, (...) dann gehe ich meinen Tee machen, dann nehme ich immer meine Tabletten, die neben dem
Kocher stehen, so vergesse ich sie nicht» (I.1: 148).

Zusätzliche Medikamente, die zur gleichen Tageszeit wie vor der Hospitalisation eingenommen werden müssen, bereiten nach Spitalaufenthalt keine Schwierigkeiten. Bei Medikamenten, die außerhalb der früheren Routine eingenommen werden müssen, ist das Erlangen der Routine wichtig. Dies gelingt rasch, wenn die neuen Medikamente mit gewohnten Alltagsaktivitäten verbunden werden können. «Das Pflaster packe ich einfach aus, am Abend, und wenn ich die Toilette gemacht habe, klebe ich es auf» (I.4: 34). Andere ältere Menschen suchen noch nach Lösungen.

Bei Unterbrechung des gewohnten Tagesrhythmus vergessen einige Befragte die Medikamente. Bemerken sie dies, nehmen sie sie umgehend ein. Andere berichten, dass sie die Medikamente noch nie vergessen haben.

\section{Unterstützung durch Ärzte/Ärztinnen und Pflegefachpersonen}

Ärzte/Ärztinnen und Pflegefachpersonen nehmen eine besondere Rolle ein, wenn es um das Erleben von Sicherheit beziehungsweise Unsicherheit im Medikamentenmanagement aus Sicht der älteren Menschen geht. Vertrauen und strukturelle Gegebenheiten spielen hier eine wichtige Rolle. Unterschiedliche Wahrnehmungen bezüglich Spital und Hausarztbetreuung verwundern daher nicht. Für die älteren Menschen ist wichtig, dass sie von den Gesundheitsfachpersonen, sei es im Spital oder vom Hausarzt/von der Hausärztin, als gleichwertige Partner(innen) im Zusammenhang mit ihrem Medikamentenregime behandelt werden. Sie nehmen die Verantwortung für ihr Medikamentenmanagement ernst. Im Spital: Mehrheitlich fühlen sich die älteren Menschen während des Spitalaufenthaltes durch die Ärzte/Ärztinnen und die Pflegefachpersonen aus- 
reichend informiert und wünschen sich nur minimale Zusatzinformationen. Bei Unklarheiten fragen manche bei den Gesundheitsfachpersonen nach und erhalten befriedigende Antworten. Einige schätzen es, als gleichwertige Partner(innen) mit Erfahrung und Wissen im Medikamentenmanagement behandelt zu werden. Andere fühlen sich vom Gesundheitspersonal im Spital nicht ernst genommen. «Ich habe gesagt, ich hätte den Mund voller Aphten und könne nichts essen. Der Doktor hat gesagt er bringe mir etwas; es ist nie gekommen: Da fühlt man sich nicht ernst genommen» (I.2: 136). Langjährige bewährte Therapien werden umgestellt, was zu starken Nebenwirkungen und Unverständnis führt. «Die Medikamente, die ich schon jahrelang genommen habe, im Spital stoppt man diese und gibt mir etwas anderes und das gibt mir dann Durchfall» (I.2: 6).

Alle erhalten ein Rezept, welches sie nicht immer als solches betrachten. «Rezept? Es war einfach ein Schreiben, das habe ich der Apotheke abgegeben» (I.1: 76). Die Informationen bei Abgabe der Austrittsdokumente sind zum Teil minimal. «Er hat einfach gesagt, es sei alles drin und es sei eine Tabelle drin mit den Tabletten» (I.3). Teilweise sind die Informationen ungenau, sodass sie die Medikamente nicht wie verordnet einnehmen. "Er [der Arzt] hat es mir nicht erklärt, er hat mir nur gesagt, wegen der Zuckerkrankheit müsse ich ein Tablettli nehmen» (I.10: 91).

Vor Austritt keine Informationen erhalten zu haben, begründen einige Befragte mit der fehlenden Anwesenheit der pflegerischen Bezugsperson. «Am Morgen kam eine neue Pflegefachfrau. $\mathrm{Ja}$, ich denke das ist darum, dass es drunter und drüber ging» (I.3: 107).

Verunsichert fühlen sich die älteren Menschen bei unterschiedlichen Informationen durch mehrere Personen und bei unklarer medikamentöser Therapie bis zum Austrittstag. «Bis am letzten Tag hieß es, ich müsse zuerst was nehmen, irgendwie noch Spritzen. Dann haben sie am letzten Tag gesagt, nein, das sollte ohne gehen» (I.9: 106). Andere haben nicht die Kraft und Aufmerksamkeit, sich Informationen $\mathrm{zu}$ merken oder einzuholen.

Einige ältere Menschen lösen das Rezept wegen Müdigkeit nicht ein und die verordnete medikamentöse Therapie kann, wenn nicht von den Angehörigen organisiert, nicht weitergeführt werden. «Ich habe das Mittel noch nicht, ich muss es noch holen gehen. Als ich entlassen wurde, ging ich nach Hause und wollte nichts als mich niederlegen» (I.6: 21). Einige Befragte packen alle Dokumente ungelesen in einen Briefumschlag und gehen direkt nach Austritt zum Hausarzt/zur Hausärztin. Andere lösen das Rezept absichtlich nicht sofort ein, weil sie zuerst mit dem Hausarzt/der Hausärztin die Therapie besprechen wollen.

Durch Hausarzt/Hausärztin: Er/sie ist die wichtigste Vertrauensperson im Zusammenhang mit dem Medikamentenmanagement. Bei Unsicherheiten mit der verordneten Therapie des Spitals lassen die älteren Menschen in der Praxis die Verordnung des Spitals auf 〈Richtigkeit〉 überprüfen. «Ja ein Betäubungsmittel, ich wollte zuerst zum Hausarzt, damit er das Rezept sieht und ich es von ihm erhalte» (I.6: 19). Sie erhalten Ratschläge und diskutieren mögliche Änderungen mit ihrem Hausarzt/ihrer Hausärztin.

\section{Diskussion}

Im Fokus dieser Studie standen das Erleben und der Umgang mit komplexen, häufig veränderten Medikamentenverordnungen nach Spitalaustritt aus der Perspektive multimorbider älterer Menschen in der Schweiz. Das Interesse lag hierbei auf dem Lebensalltag im häuslichen Kontext in den ersten vier- zehn Tagen nach dem Spitalaufenthalt. Die Ergebnisse zeigen, dass für ältere Menschen das Medikamentenmanagement viele Aspekte beinhaltet: die subjektive Krankheitswahrnehmung (Multimorbidität), die Einstellung zur Wirkung und zum Nutzen ihrer Medikamente, ihr Verständnis zur Verantwortungsverteilung bezüglich des Medikamentenmanagements, das Entwickeln und Umsetzen von Strategien zum Medikamentenmanagement sowie die Rollenwahrnehmung von Ärzten/Ärztinnen und Pflegefachpersonen in Bezug auf Medikamententherapien. Durch alle Kategorien zieht sich das Bedürfnis nach dem Erhalt der Selbstständigkeit. Folgt man den Ausführungen von Graefe, van Dyk und Lessenich (2011) zu «Alter(n)snormen und Selbstkonzepten» in der Lebensphase nach dem Erwerbsleben, so verwundert dies nicht. Demnach wird das passive Rentendasein verdrängt durch den Wunsch nach einer aktiven, selbstregulierenden Lebensphase. «Betroffene» nehmen sich selbst vor allem als «aktive und normale Erwachsene» ohne spezifische Bedürfnisse oder Einschränkungen wahr (Graefe et al., 2011). Erst der Übergang ins abhängige Alter wird von ihnen häufig als Einbruch ihrer Autonomie erlebt (Graefe et al., 2011). Für einige ältere Menschen in der vorliegenden Studie wäre der Verlust der Autonomie so schlimm, dass sie, um nicht von Dritten abhängig zu sein, auf die Medikamente verzichten würden. Multimorbidität und die Einnahme einer Vielzahl von Medikamenten werden, solange sie diese selbstständig managen können, entsprechend nicht als einschneidend erlebt. Auch Lumme-Sandt und Virtanen (2002) bestätigen, dass ältere Menschen, im Gegensatz zur weitverbreiteten Meinung der Gesundheitsfachpersonen, im Zusammenhang mit ihrem Medikamentenmanagement sehr wohl eine aktive Rolle einnehmen. Sie schät- 
zen es, als gleichwertige Partner mit ihrem Wissen in die Behandlung einbezogen zu werden.

Für die hier Befragten ist das Bewahren der Selbstständigkeit im Alter trotz Multimorbidität sehr wichtig. Für sie stellt das Krankheits- und Medikamentenmanagement einen wichtigen Teil des Umgangs mit der Multimorbidität dar, wie es auch die multimorbiden Befragten mittleren Alters bei Townsend, Hunt und Wyke (2003) beschreiben. Im Gegensatz zu den befragten chronisch Kranken bei Haslbeck (2008) äußern die älteren Menschen hier nicht den Wunsch nach einem normalen Leben ohne Medikamente. Die Befragten dieser Studie akzeptieren Einschränkungen in ihrem Alltagsleben durch Krankheiten und Medikamenteneinnahme als zum Alter gehörend, wie es auch Kippen et al. (2005) beschreiben. Das eigenständige Management ihrer Krankheit und der Medikamente ist für sie unabdingbar, sie vertrauen hinsichtlich ihrer medikamentösen Therapie vor allem sich selbst. Die Angst vor Fehlern durch Dritte aber auch das Bedürfnis, die Verantwortung für potenzielle Fehler selbst tragen zu wollen, beeinflussen diese Haltung.

Um ihre Selbstständigkeit zu erhalten, entwickeln die älteren Menschen Mechanismen und Routinen, die sie dabei unterstützen. Auch Swanlund et al. (2008) beschreiben das Entwickeln oder Anpassen von Gewohnheiten, Gedächtnisstützen, Erinnerungshilfen und Routinen rund um das Medikamentenmanagement als erfolgreiche Strategien. Die älteren Menschen in der vorliegenden Studie entwickeln alle ein individuelles System für das Richten der Medikamente, das die Routine in der Einnahme unterstützt. Diesen essenziellen Vorgang beschreiben verschiedene Studien (Haslbeck \& Schaeffer, 2009, Reid et al., 2005; Swanlund et al., 2008). Wichtig ist, dass die Medikamenteneinnahme an eine Alltagstätig- keit gekoppelt und so zur Routine wird. Im Gegensatz zu Haslbeck und Schaeffer (2009), die beschreiben, dass in der Routine auch die Gefahr besteht, die Medikamenteneinnahme $\mathrm{zu}$ vergessen, weisen die älteren Menschen hier darauf hin, dass diese Routine unabdingbar sei, um die Medikamente korrekt einnehmen zu können. Eine mögliche Erklärung ist, dass die älteren Menschen, im Gegensatz zu den Befragten bei Haslbeck und Schaeffer (2009), alle pensioniert sind und wie von Kuhlmey (2009) beschrieben, Gesundheit für ältere Menschen der wichtigste Lebensbereich und somit die Medikamenteneinnahme ein Beitrag zur Stabilisierung der Gesundheit ist.

Müssen Medikamente nach Spitalaustritt $\mathrm{zu}$ neuen Zeiten eingenommen werden, suchen die älteren Menschen nach einer neuen Routine. Haslbeck (2008) schildert dies als Komplexitätssteigerung, was für die Patient(inn)en in hohem Maß irritierend sein kann. In der vorliegenden Studie wird diese Verunsicherung wenig beobachtet. Ein viel größeres Problem entsteht durch die Veränderung der Medikamente im Umgang mit dem Wochendispenser, da häufig zum Verwechseln ähnlich aussehende Medikamente ausgetauscht werden müssen. $\mathrm{Zu}$ dieser Problematik, Medikamentenänderung in Kombination mit Einsatz von Wochendispenser, konnten in der Literatur keine Hinweise gefunden werden.

Die älteren Menschen nennen Namen und Wirkung der Medikamente, auch von den bei Spitalaustritt neu verordneten, was im Unterschied zur Befragung von Patient(inn)en mit Herzinsuffizienz von Reid et al. (2005) steht. Im Gegensatz zu den Befürchtungen von Pruszydlo et al. (2008), die Umstellung von angestammten Medikamenten auf Generika sei für die Betroffenen verwirrend und könne zu Fehlern führen, betonen die älteren Menschen der vorliegenden Studie, dass die Umstel- lung für sie kein Problem darstellt. Diese Sicherheit könnte durch die jahrelange informierte und routinierte Medikamenteneinnahme der Befragten begründet sein.

Die Informationsweitergabe durch die Ärzte/Ärztinnen und Pflegefachpersonen wird von den älteren Menschen unterschiedlich wahrgenommen. Einerseits scheinen Informationen durch die Gesundheitsfachpersonen zu veränderten Medikamentenplänen aus Sicht der multimorbiden älteren Menschen nicht immer ausreichend oder zum falschen Zeitpunkt zu erfolgen. Andererseits fühlen sich ältere Menschen gut über die medikamentöse Therapie nach Spitalaustritt informiert, was im Gegensatz zu den Ergebnissen von Knight et al. (2011) steht. Eine mögliche Erklärung ist, dass die Befragten dieser Studie ein enges Vertrauensverhältnis zum Hausarzt/zur Hausärztin schildern und Fragen zur medikamentösen Therapie des Spitals durch ihn/sie beantwortet werden. Modig, Kristensson, Troein, Brorsson und Midlöv (2012) bestätigen in ihrer Studie zu Erfahrungen mit Informationen zu Medikamenten von älteren Menschen, dass das Vertrauen in den Arzt/die Ärztin entscheidend ist, ob ein älterer Mensch sich ausreichend über die Medikamente (Einnahme, Wirkungen, Nebenwirkungen) informiert fühlt. Wissen über Wirkungen, Nebenwirkungen und Einnahme ihrer Medikamente sind aus Sicht der Betroffenen eine wichtige Voraussetzung für ein selbstständiges Medikamentenmanagement. Auch Lumme-Sandt und Virtanen (2002) bestätigen, dass ältere Menschen, im Gegensatz zur weitverbreiteten Meinung der Gesundheitsfachpersonen, im Zusammenhang mit ihrem Medikamentenmanagement sehr wohl eine aktive Rolle einnehmen. Sie schätzen es, als gleichwertige Partner mit ihrem Wissen in die Behandlung einbezogen $\mathrm{zu}$ werden. Hierbei 
variiert allerdings das individuelle Bedürfnis nach Wissen, was auch Borgsteede et al. (2011) in der Studie zum Informationsbedürfnis $\mathrm{zu}$ Medikamenten bei Austritt von Erwachsenen aufzeigen. Einige Befragte wünschen sich kurze Informationen, andere eine detaillierte Aufklärung zu Nebenwirkungen. Modig et al. (2012) beschreiben hierzu zwei «Pole»: sich mit vorhandenen Informationen komfortabel fühlen und sich unsicher und ängstlich fühlen. Ältere Menschen tendieren insbesondere zum ersten Pol, wenn Vertrauen zum Hausarzt besteht und subjektiv genug Informationen verfügbar sind beziehungsweise sie sich gut informiert fühlen. Wenn diese Aspekte nicht gegeben sind, fühlen die Betroffenen sich eher unsicher bezogen auf ihr Medikamentenmanagement.

Oft wird Kranken von Gesundheitsfachpersonen die alleinige Verantwortung für ihr Medikamentenmanagement und entsprechend auch für das Versagen der medikamentösen Therapie angelastet (Kamche \& Haslbeck, 2004). Die vorliegende Studie zeigt aber auch, dass Informationen der Gesundheitsfachpersonen zur medikamentösen Therapie oft nicht der Lebenssituation der Patient(inn)en angepasst sind, zum falschen Zeitpunkt vermittelt werden und nicht nachgefragt wird, wie der Patient/die Patientin nach Austritt die neu verordneten Medikamente organisieren kann. Im Gegensatz zur Studie von Knight et al. (2011) in England erhalten die älteren Menschen in der Schweiz bei Spitalaustritt keine Medikamente mit nach Hause. Können weder die Betroffenen noch die Angehörigen das Rezept einlösen oder erfolgt der als relevant erachtete Einbezug des Hausarztes/der Hausärztin zeitlich verzögert, kommt es zur Unterbrechung der verordneten medikamentösen Therapie.

Zusammenfassend zeigen die Ergebnisse, dass Multimorbidität und Mehr- fachmedikation im Alter mit dem Wunsch nach Selbstständigkeit vereinbar sind. Der Erhalt ihrer Selbstständigkeit im Medikamentenmanagement kann älteren Menschen helfen, sich selbst weiterhin im Sinne von Graefe et al. (2011) als aktive Personen zu sehen. Das Entwickeln und Wiedererlangen von neuen Alltagsroutinen nach Veränderungen im komplexen Medikamentenmanagement hat entsprechend einen hohen Stellenwert. Hierdurch gelingt es ihnen, das Medikamentenmanagement in ihre Alltagsnormalität zu integrieren.

\section{Limitationen}

Eine methodologische Limitation der Studie ist die kleine und heterogene Untersuchungsgruppe. Dies zeigte sich unter anderem in der Altersspanne und der Anzahl der einzunehmenden Medikamente. Bei einigen Interviews war der Partner/die Partnerin anwesend, was möglicherweise das Antwortverhalten der Teilnehmenden beeinflusste.

Ein zentrales Ergebnis dieser Studie ist die Bedeutung, die der Verantwortung im Zusammenhang mit dem Medikamentenmanagement beigemessen wird. Da nur ältere Menschen befragt wurden, die ihr Medikamentenmanagement selbstständig durchführten, ist dies auf den ersten Blick nicht verwunderlich. Andere Studien zeigen jedoch auf, dass ältere Menschen gerne auf die informelle familiäre Unterstützung im Krankheitsmanagement zurückgreifen. Für einige Befragte dieser Studie ist die Abgabe der Verantwortung für das Medikamentenmanagement an Dritte kaum denkbar. Weiterführende Aspekte zu möglichen Befürchtungen und Erfahrungen in diesem Zusammenhang wurden nicht befragt. Dies müsste Gegenstand einer weiteren Studie sein. Auch wäre zu explorieren wie ältere Menschen, die die Unterstützung durch Angehörige oder Gesundheitsfachpersonen benötigen, ihre Eigenverantwortung und Autonomie im Zusammenhang mit dem Medikamentenmanagement erleben. Erfahrungen der Gesundheitsfachpersonen und Angehörigen wurden nicht erfragt, was eine Ergänzung zur Sicht der älteren Menschen ergeben hätte. Case-Studies könnten hier ergänzend wichtige Erkenntnisse liefern.

Die befragten älteren Menschen erleben sich als sicher im Umgang mit ihren Medikamenten. Ob eine Diskrepanz zwischen der subjektiven Selbsteinschätzung und dem realen Umgang bestand, wurde im Rahmen dieser Studie nicht erfasst.

Da alle Interviewten in derselben Klinik hospitalisiert waren, bleibt die Frage offen, ob die Ergebnisse auf andere Kliniken übertragbar sind. Hierzu bedarf es weiterer Untersuchungen.

Dennoch gibt diese Studie erste Hinweise auf das Erleben und den Umgang von älteren Menschen mit komplexen Medikamentenverordnungen nach Spitalaustritt.

Um die Güte der Studie zu sichern, erfolgten verschiedene Maßnahmen: Die Glaubwürdigkeit der Datenanalyse wurde erhöht, indem die Ergebnisse neun Studienteilnehmenden vorgelegt wurden. Diese bestätigten alle die Resultate, was Lincoln und Guba (1985) als wichtigstes Gütekriterium beschreiben. Weiter wurden die einzelnen Analyseschritte und vorläufige Resultate mit Mentorin und Studierenden kritisch diskutiert und die einzelnen Analyseschritte dokumentiert.

\section{Schlussfolgerungen}

Die Studie zeigt, dass der Prozess des Medikamentenmanagements in der Zeit nach Spitalaustritt aus Sicht der Betroffenen vor allem durch das Be- 
streben, die Selbstständigkeit zu erhalten bzw. wiederzugewinnen, geprägt ist. Hierbei stoßen sie jedoch zum Teil an Grenzen. Die Ergebnisse weisen darauf hin, dass Unregelmäßigkeiten in der Medikamenteneinnahme in der Zeit nach dem Spitalaustritt durch Einschnitte im bisher selbstständigen Medikamentenmanagement ausgelöst werden können. Mangelhafte Informationen und fehlendes Hinterfragen von Alltagsgewohnheiten im Zusammenhang mit dem Medikamentenmanagement durch Gesundheitsfachpersonen im Spital können zu Unregelmäßigkeiten in der Medikamenteneinnahme nach dem Austritt führen. Um die Kontinuität über die Spitalzeit hinaus zu fördern, werden lebensweltnahe Konzepte zum Medikamentenregime benötigt, die bereits während des Spitalaufenthaltes ansetzen. Es gilt eine Sensibilität für den individuellen Bedarf der älteren Menschen zu entwickeln, um Therapie und selbstständige Umsetzung im Alltag zu Hause aufeinander abzustimmen. Das Besprechen der Therapie anhand des Rezepts, das Nachfragen, wie nach Austritt die Medikamente beschafft werden können, und/oder ob ein erster Termin mit dem Hausarzt/der Hausärztin abgemacht wurde, sollte beispielsweise zum selbstverständlichen Inhalt von Austrittsgesprächen werden. Pflegefachpersonen im Spital können hier eine Schlüsselfunktion einnehmen, indem sie durch eine kompetente Erfassung der Bedürfnisse und patientenbezogene Beratungen die älteren Menschen unterstützen, ihr Medikamentenmanagement auch zu Hause weiterhin selbstständig und korrekt durchzuführen.

Konkrete Unterstützungsangebote zur Entwicklung oder Förderung von neuen Routinen bei veränderten Medikamentenverordnungen nach Spitalaufenthalten fehlen bislang. Hier zeigt sich weiterer Untersuchungs- und Entwicklungsbedarf.

\section{Danksagung}

Wir danken allen Betagten für die ehrlichen und interessanten Gespräche und für die Offenheit, die Interviews bei ihnen zu Hause durchführen zu können. Den Studienkolleg(inn)en der Berner Fachhochschule für Gesundheit danken wir für die wertvollen Diskussionen und Unterstützungen bei der Analyse.

\section{Beitrag der Autorinnen}

Planung, Datenerhebung und -analyse, Verfassen des Manuskripts: YS; Begleitung bei Planung und beim Verfassen des Manuskripts: ES; Projektverantwortung, Planung, Begleitung im Analyseprozess und beim Verfassen des Manuskripts: CM

\section{Taking a multitude of medications} after patient discharge - a qualitative study of the experiences of older people with multimorbidity Background: Older people often have multimorbidity requiring a complex regime of medications, which may change following hospital discharge, thus presenting new challenges. The experiences of older people, who manage their own medications, in particular following hospital discharge, have rarely been studied.

Aim: This study investigates the experiences of older people with multimorbidity taking multiple medications after hospital discharge and how they cope with medication-taking.

Method: A qualitative-descriptive approach with ten interviews was chosen. The data were coded openly into two groups according to Saldaña (2013).
Results: It is important for older people, in spite of their multimorbidity, to maintain their independence and maintain responsibility for taking their medications. Routines are developed from taking medications over many years and the new medications are easily integrated. Unclear information by the medical staff and the inability of the older people to obtain the medications after discharge may lead to mistakes or interruptions in the drug therapy at home. The key person for this group is the general practitioner, even concerning the drug therapy initiated in hospital.

Conclusion: It is advisable to adapt discharge education to the needs of older people, especially with regard to their drug therapy, to its integration into their daily routine, and to any possible shortcomings in their medication management.

Key words: Older persons, polypharmacy, self-management, medication management following discharge, qualitative research

\section{Literatur}

Banning, M. (2009). A Review of interventions used to improve adherence to medication in older people. International Journal of Nursing Studies, 46 (11), $1505-1515$.

Borgsteede, S. D.; Karapinar-Çarkit, F.; Hoffmann, E.; Zoer, J.; van den Bemt, P. M. L. A. (2011). Information needs about medication according to patients discharged from a general hospital. Patient Education and Counselling, 83 (1), $22-28$.

Boyd, C. M.; Fortin, M. (2010). Future of multimorbidity research: How should understanding of multimorbidity in- 
form health system design? Public Health Reviews, 32 (2), 451 - 474.

Bundesamt für Statistik (2010). Szenarien zur Bevölkerungsentwicklung der Schweiz 2010-2060. http://www.bfs. admin.ch/bfs/portal/de/index/news/ puplikationen.html?publicationID $=3989$ [27.01.2014].

Bundesamt für Statistik (2014a). Bevölkerungsdaten im Zeitvergleich, am Ende des Jahres. http://www.bfs.admin.ch/ bfs/portal/de/index/themen/01/02/ blank/key/alter/gesamt.html [27.01.2014].

Bundesamt für Statistik (2014b). Krankenhausfälle, Patienten und Aufenthaltsdauer, 2012. http://www.bfs.admin.ch/ bfs/portal/de/index/themen/14/ 04/01/key/inanspruchnahme.html [27.01.2014].

Creshwell, J. W. (2007). Qualitative Inquiry and Research Design: Choosing Among Five Approaches (2. ed.). London: Sage.

DeBartolomeo Mager, D.; Madigan, E. A. (2010). Medication use among older adults in a home care setting. Home Healthcare Nurse, 28 (1), 14-21.

De Geest, S.; Abraham, I.; Gemoets, H.; Evers, G. (1994). Development of the long-term medication behaviour selfefficacy scale: Qualitative study for item development. Journal of Advanced Nursing, 19 (2), 233-238.

Flick, U. (2011). Qualitative Sozialforschung, Eine Einführung. (4 Aufl.). Reinbek bei Hamburg: Rowohlt.

Foust, J. B.; Vuckovic, N.; Henriquez, E. (2012). Hospital to home health care transition: patient, caregiver, and clinician perspectives. Western Journal of Nursing Research, 34 (2), 194-212.

Gläser, J.; Laudel, G. (2009). Experteninterviews und qualitative Inhaltsanalyse (3 Aufl.). Wiesbaden: VS Verlag für Sozialwissenschaften.

Gordon, K.; Smith, F.; Dhillon, S. (2007). Effective chronic disease management: patients' perspectives on medicationrelated problems. Patient Education and Counselling, 65 (3), $407-415$.

Göckenjan, G. (2010). Altersbilder in der Geschichte. In: Aner, K.; Karl, U. (Hrsg.). Handbuch Soziale Arbeit und Alter. Wiesbaden: VS Verlag für Sozialwissenschaften, $408-413$.
Graefe, S.; van Dyk, S.; Lessenich, S. (2011). Altsein ist später. Zeitschrift fur Gerontologie und Geriatrie, 44 (5), 299-305.

Häfeli, W. E. (2011). Polypharmazie. Schweizer med Forum, 11 (47), 847 - 852.

Haslbeck, J. (2008). Bewältigung komplexer Medikamentenregime aus Sicht chronisch Kranker. Pflege \& Gesellschaft, 13 (1), 48-61.

Haslbeck, J.; Schaeffer, D. (2009). Routines in medication management: The perspective of people with chronic conditions. Chronic Illness, 5 (3), 184- 196.

Kamche, A.; Haslbeck, J. (2004). Komplexe Medikamententherapien bewältigen, theoretische Betrachtung einer Herausforderung im Lebensalltag chronisch kranker Menschen. Pflege \& Gesellschaft, 9 (4), 147 - 153.

Kippen, S.; Fraser, M.; Ellis, J. (2005). As time goes by: Issues for older people with their medication use. Australasian Journal on Ageing, 24 (2), 103-107.

Knight, D. A.; Thompson, D.; Mathie, E.; Dickinson, A. (2011). Seamless care? Just a list would have helped! Older people and their carerss experiences of support with medication on discharge home from hospital. Health Expectations, 12 (2), 1- 15.

Kuhlmey, A. (2009). Chronische Krankheit in der Lebensphase Alter. In: Schaeffer, D. (Hrsg.). Bewältigung chronischer Krankheit im Lebenslauf. Bern: Hans Huber.

Laugaland, K.; Aase, K.; Barach, P. (2012). Interventions to improve patient safety in transitional care - a review of the evidence. Work - Journal of Prevention Assessment and Rehabilitation, 41 (1), $2915-2924$.

Lincoln, Y.; Guba, E. G. (1985). Naturalistic Inquiry. London: Sage Publications.

Ludwig, A. (2005). Herausforderungen komplexer Medikamentenregime bei chronischen Erkrankungen. Magister, Universität Bielefeld Bielefeld. http:// www.uni-bielefeld.de/gesundhw/ag6/ pdv/projekte/B2.html [08.03.2013].

Lumme-Sandt, K.; Virtanen, K. (2002). Older people in the field of medication. Sociology of Health \& Illness, 24 (3), $285-304$.

Marengoni, A.; Angleman, S.; Melis, R.; Mangialasche, F.; Karp, A.; Garmen, A.;
Meinow, B.; Fratiglioni, L. (2011). Aging with multimorbidity: A systematic review of the literature. Ageing Research Reviews, 10 (4), 430 - 439.

Miles, M. B.; Huberman, A. M. (1994). Qualitative Data Analysis: A Sourcebook of New Methods. (2. ed.). Beverly Hills, CA: Sage.

Modig, S; Kristensson, J.; Troein, M.; Brorsson, A.; Midlöv, P. (2012). Frail elderly patients〉 experiences of information on medication. A qualitative study. BMC Geriatrics, 12, 46. doi: 10.1186/ 1471-2318-12-46 [27.01.2014].

Moen, J.; Bohm, A.; Tillenius, T.; Antonov, K.; Nilsson, L. L. G.; Ring, L. (2009). “I don't know how many of these [medicines] are necessary." - A focus group study among eldery users of multiple medicines. Patient Education and Counselling, 74 (2), 135 - 141.

Müller-Bühl, W.; Gerold, C.; Engeser, P.; Szecsenyi, J. (2009). Changes in drug therapy following hospital discharge for patients in a general pratice: A german incident study. Journal of Public Health, 17 (3), 212 - 223.

Neuner-Jehle, S. (2011). Zuviel des Guten Rezepte gegen Polypharmazie. Primary Care, 11 (12), 212- 215.

Pruszydlo, M. G.; Quinzler, R.; Kaltschmidt, J.; Haefeli, W. E. (2008). Medizinische Probleme und Risiken bei der rabattvertragsgerechten Umstellung von Medikationen in Deutschland. Deutsche Medizinische Wochenschrift, 133 (27), $1423-1428$.

Reid, M.; Clark, A.; Murdoch, D. L.; Morrison, C.; Capewell, S.; McMurray, J. (2005). Patients strategies for managing medication for chronic heart failure. International Journal of Cardiology, 109 (1), $66-73$.

Ritchie, J.; Lewis, J. (2003). Qualitative Research Practice. A Guide for Social Science Students and Researchers. London: Sage.

Ryan, R.; Santesso, N.; Hill, S.; Lowe, D.; Kaufmann, C.; Grimshaw, J. (2012). Consumer-oriented interventions for evidence based prescribing and medicines use: An overview of systematic reviews. The Cochrane Library (2). doi: 10.1002/14651858.CD007768.pub2 [08.04.2013]. 


\section{Originalarbeit}

Saldaña, J. (2013). The Coding Manual for Qualitative Researchers (2 ed.). London: Sage.

Sandelowski, M. (2010). What ss in a name? Qualitative description revisited. Research in Nursing \& Health, 33 (1), $77-84$.

Swanlund, S. L.; Scherck, K. A.; Metcalfe, S. A.; Jeske-Hale, S. R. (2008). Keys to succsessful self-management of medications. Nursing Science Quarterly, 21 (238), 238-246.

Townsend, A.; Hunt, K.; Wyke, S. (2003). Managing multiple morbidity in mid-life: A qualitative study of attitudes to drug use. British Medical Journal, 327 (7419), $1-6$.

Wurm, S.; Wiest, M.; Tesch-Römer, C. (2010). Theorien zu Atern(n) und Gesundheit. Bundesgesundheitsblatt - Gesundheitsforschung - Gesundheitsschutz, 53 (5), $498-503$.

Ybanez-Blomstrom, E. M. (2008). The Essence of the Experience of Polypharmacy in the Life-World of the Communitiy Dwelling Elder. Doctor of Philosophy, Texas, Galveston. http://udini.proquest. com/view/the-essence-of-the-experience-of-goid:304374272/. [08.04.2013].
Was war die größte Herausforderung bei Ihrer Studie?

Die älteren Menschen zu ermutigen, über ihre Erfahrungen mit den vom Gesundheitspersonal erhaltenen Informationen zum Medikamentenmanagement zu erzählen.

\section{Was wünschen Sie sich bezüglich der Thematik für die Zukunft?}

Gesundheitspersonal, das ältere Menschen, die ihr Medikamentenregime selbstständig durchführen, nach Bedürfnissen bezüglich des Medikamentenmanagements befragt und sie angepasst informiert.

\section{Was empfehlen Sie den LeserInnen zum Weiterlesen/Vertiefen?}

Haslbeck, J. (2009). Medikamente und chronische Krankheit. Bern: Hans Huber.

\section{Korrespondenzadresse}

Yvonne Stauffer

Inselspital, Universitätsspital Bern

Universitätsklinik für Allgemeine Innere Medizin

3010 Bern

Schweiz

yvonne.stauffer@insel.ch 